\title{
Running Spectral Index and Formation of Primordial Black Hole in Single Field Inflation Models
}

\author{
Manuel Drees* and Encieh Erfani ${ }^{\dagger}$ \\ Physikalisches Institut and Bethe Center for Theoretical Physics, Universität Bonn, \\ Nussallee 12, 53115 Bonn, Germany
}

प प

\begin{abstract}
A broad range of single field models of inflation are analyzed in light of all relevant recent cosmological data, checking whether they can lead to the formation of long-lived Primordial Black Holes (PBHs). To that end we calculate the spectral index of the power spectrum of primordial perturbations as well as its first and second derivatives. $\mathrm{PBH}$ formation is possible only if the spectral index increases significantly at small scales, i.e. large wave number $k$. Since current data indicate that the first derivative $\alpha_{S}$ of the spectral index $n_{S}\left(k_{0}\right)$ is negative at the pivot scale $k_{0}, \mathrm{PBH}$ formation is only possible in the presence of a sizable and positive second derivative ("running of the running") $\beta_{S}$. Among the three small-field and five large-field models we analyze, only one smallfield model, the "running mass" model, allows PBH formation, for a narrow range of parameters. We also note that none of the models we analyze can accord for a large and negative value of $\alpha_{S}$, which is weakly preferred by current data.
\end{abstract}

*drees@th.physik.uni-bonn.de

†erfani@th.physik.uni-bonn.de 


\section{Introduction}

An epoch of accelerated expansion in the early universe, inflation, dynamically resolves many cosmological puzzles of the hot big bang model, such as homogeneity, isotropy and flatness of the Universe [1]. On the other hand, the generation of a spectrum of primordial fluctuations in the early universe is a crucial ingredient of all inflationary models. These fluctuations can explain the generation of all (classical) inhomogeneities that can be seen in our universe, from the Cosmic Microwave Background (CMB) anisotropies to the Large Scale Structure (LSS) in the form of (clusters of) galaxies [2]. The inflationary paradigm therefore reconciles Big Bang cosmology with the appearance of an inhomogeneous universe. In addition, many models of inflation [3] make accurate predictions allowing for observations of ever increasing variety and quality to discriminate between the various candidates.

One such prediction concerns the possible formation of Primordial Black Holes (PBHs). Indeed, it was realized already some time ago that the spectrum of primordial fluctuations could lead to the production of PBHs [4, 5]. For this generation mechanism to be efficient, one typically needs a "blue" spectrum [6, 7]. This means that the amplitude of density fluctuations must be much higher at the small length scales relevant for $\mathrm{PBH}$ formation than at the much larger length scales probed by data on the Cosmic Microwave Background (CMB) and Large Scale Structure (LSS). This discrepancy of scales makes PBH formation also a unique probe of cosmological inflation; in particular, the constraint that not too many PBHs should have been produced has been used to limit the power spectrum at small length scales, which in turn allows to constrain models of inflation [8, 9]. For single-field inflation models, the relevant parameter space is defined by the scalar spectral index $n_{S}$; the ratio of tensor to scalar fluctuations $r$; the running of the scalar spectral index $\alpha_{S}$; and we will see that the "running of running of the spectral index", $\beta_{S}$, is also important for PBH formation.

The goal of this paper is to make use of the recent observational bounds on these parameters derived from the combined CMB data from the Wilkinson Microwave Anisotropy Probe seven year data (WMAP7) [10] and the South Pole Telescope (SPT) [11], as well as data on the Baryon Acoustic Oscillations (BAO) [12], the Hubble constant $H_{0}$ [13], and clusters of galaxies [14]. We will investigate a wide range of inflationary models, checking whether they still can give rise to significant $\mathrm{PBH}$ formation given these constraints. In so doing, we also check whether these models can account for a sizably negative running of the spectral index, as (weakly) favored by current data. We focus on models where the cosmic expansion was driven by a single, selfinteracting scalar inflaton field $\phi$. Moreover, we only consider models with simple potentials, which have been suggested for reasons not related to PBH formation.

This paper is organized as follows: In section 2 we present a brief review of the PressSchechter formalism [15] describing PBH formation. In section 3 we first summarize the current bounds on the observational parameters, and review their calculation from the potential of the inflaton field. We then systematically analyze three small-field and five large-field models. In section 4 we present our conclusions. 


\section{Primordial Black Holes Formation}

PBHs are black holes that result from the collapse of density fluctuations [16, 17]. They are very sensitive cosmological probes for physics phenomena occurring in the early universe. They could be formed by many different mechanisms, e.g., from initial density inhomogeneities [16], a softening of the equation of state [18] (e.g. in phase transition or during the preheating period after inflation), collapse of cosmic string loops [19], bubble collisions [20, collapse of domain walls [21], etc.

The idea that large amplitude matter overdensities in the early universe could have collapsed through self-gravity to form PBHs was first studied by Zel'dovich and Novikov [16], and then by Hawking [17]. This theory suggests that large amplitude inhomogeneities in the early universe overcome internal pressure forces and collapse to form black holes. A lower threshold for the amplitude of such homogeneities $\delta_{\text {th }} \equiv(\delta \rho / \rho)_{\text {th }}$, was first provided by Carr [5], giving $\delta_{\text {th }} \approx 1 / 3$ at the time of radiation domination (RD) 1 The probability of $\mathrm{PBH}$ formation is a useful tool to constrain the mean amplitude of inhomogeneities on scales which cannot be probed by any other method. Since PBHs behave like matter, their contribution to the energy density increases with time during the RD epoch. For this reason, the PBHs formed considerably before the end of RD are the most relevant to cosmology. We have focused in our study [7] on these kind of PBHs and we also considered the standard case of PBHs formation, which applies to scales which have left the horizon at the end of inflation ${ }^{2}$ We only consider Gaussian and spherically symmetric perturbations and we assume that the mass of the $\mathrm{PBH}$ formed is proportional to the mass of the horizon mass at horizon entry, $M_{\mathrm{PBH}}=\gamma M_{\mathrm{PH}} 3^{3}$

Generally, for Gaussian primordial fluctuations, the probability density $P(\delta ; R)$, where $\delta$ is the density contrast averaged over a sphere of radius $R$, is given by

$$
P(\delta ; R)=\frac{1}{\sqrt{2 \pi} \sigma_{\delta}(R)} \exp \left(-\frac{\delta^{2}}{2 \sigma_{\delta}^{2}(R)}\right) .
$$

Here, the dispersion (mass variance) $\sigma_{\delta}(R)$ is computed using a Gaussian window function $W(k R)=\exp \left(-\frac{k^{2} R^{2}}{2}\right):$

$$
\sigma_{\delta}^{2}(R)=\int_{0}^{\infty} W^{2}(k R) \mathcal{P}_{\delta}(k) \frac{\mathrm{d} k}{k},
$$

where $\mathcal{P}_{\delta}(k)$ is the power spectrum of $\delta$ which is related to the power spectrum of curvature perturbations on comoving hypersurfaces as follows [2]:

$$
\mathcal{P}_{\delta}(k, t)=\frac{4(1+w)^{2}}{(5+3 w)^{2}}\left(\frac{k}{a H}\right)^{4} \mathcal{P}_{\mathcal{R}_{c}}(k) .
$$

Therefore the probability $f(\geq M)$ that a PBH with mass $\geq M$ is formed on a scale $R$ when

\footnotetext{
${ }^{1}$ Niemeyer and Jedamzik [22] carried out numerical simulations and found the threshold for PBH formation to be 0.7 . We have shown [7 that PBHs abundance is sensitive to the value of $\delta_{\text {th }}$.

${ }^{2}$ It has been shown [9] that PBHs can also form on scales which never leave the horizon during inflation, and therefore never become classical. We do not consider this contribution.

${ }^{3} \mathrm{~A}$ simple analytical calculation suggest that $\gamma \simeq w^{3 / 2} \simeq 0.2$ during the radiation era [5].
} 
that scale reenters the Hubble radius, is given by

$$
f(\geq M)=2 \gamma \int_{\delta_{\mathrm{th}}}^{\delta_{\mathrm{cut}}} P(\delta ; M(R)) \mathrm{d} \delta .
$$

In order to complete the calculation we just need to relate the PBH mass $M$ to the comoving smoothing scale $R$ when the scale enters the horizon, $R=(a H)^{-1}$. It is straightforward to show that in the RD era

$$
\frac{R}{1 \mathrm{Mpc}}=5.54 \times 10^{-24} \gamma^{-\frac{1}{2}}\left(\frac{M_{\mathrm{PBH}}}{1 \mathrm{~g}}\right)^{1 / 2}\left(\frac{g_{*}}{3.36}\right)^{1 / 6},
$$

where $g_{*}$ is the effective number of relativistic degrees of freedom which is expected to be of order 100 in the early universe.

The power spectrum of primordial scalar fluctuations at scales $k \simeq k_{R}$ is typically parameterized as a power-law with power $n_{S}, \mathcal{P}_{\mathcal{R}_{c}}(k)=\mathcal{P}_{\mathcal{R}_{c}}\left(k_{R}\right)\left(k / k_{R}\right)^{n_{S}(R)-1}$, with $k_{R}=1 / R$. With this ansatz, the variance of the primordial density field at horizon crossing is given by

$$
\sigma_{\delta}^{2}(R)=\frac{2(1+w)^{2}}{(5+3 w)^{2}} \mathcal{P}_{\mathcal{R}_{c}}\left(k_{R}\right) \Gamma\left[\left(n_{S}(R)+3\right) / 2\right],
$$

for $n_{S}(R)>-3$.

In order to relate the scales relevant for $\mathrm{PBH}$ formation to the scales probed by data on the CMB and LSS, we parameterize the power spectrum as

$$
\mathcal{P}_{\mathcal{R}_{c}}\left(k_{R}\right)=\mathcal{P}_{\mathcal{R}_{c}}\left(k_{0}\right)\left(k_{R} / k_{0}\right)^{n(R)-1} .
$$

It is important to distinguish between $n_{S}(R)$ and $n(R)$ at this point. $n_{S}(R)$ describes the slope of the power spectrum at scales $k \sim k_{R}=1 / R$, whereas $n(R)$ fixes the normalization of the spectrum at $k_{R} \gg k_{0}$. The two powers are identical if the spectral index is strictly constant, i.e. if neither $n_{S}$ nor $n$ depend on $R$. However, in this case CMB data imply [10] that $n=n_{S}$ is close to unity. Eqs. (2.6) and (2.7) then give a very small variance, leading to essentially no $\mathrm{PBH}$ formation.

Significant PBH formation can therefore only occur in scenarios with running spectral index [23]. We parameterize the scale dependence of $n$ as [24]:

$$
n(R)=n_{S}\left(k_{0}\right)-\frac{1}{2 !} \alpha_{S} \ln \left(k_{0} R\right)+\frac{1}{3 !} \beta_{S} \ln ^{2}\left(k_{0} R\right)+\ldots ;
$$

recall that we are interested in $R \ll 1 / k_{0}$, i.e. $\ln \left(k_{0} R\right)<0$. The parameters $\alpha_{S}$ and $\beta_{S}$ denote the running of the effective spectral index $n_{S}$ and the running of the running, respectively:

$$
\begin{aligned}
n_{S}\left(k_{0}\right) & \left.\equiv \frac{d \ln \mathcal{P}_{\mathcal{R}_{c}}}{d \ln k}\right|_{k=k_{0}}, \\
\alpha_{S}\left(k_{0}\right) & \left.\equiv \frac{d n_{S}}{d \ln k}\right|_{k=k_{0}}, \\
\beta_{S}\left(k_{0}\right) & \left.\equiv \frac{d^{2} n_{S}}{d \ln ^{2} k}\right|_{k=k_{0}} .
\end{aligned}
$$

\footnotetext{
${ }^{4}$ We will show that in practice $P(\delta ; R)$ is such a rapidly decreasing function of $\delta$ above $\delta_{\text {th }}$ that the upper cutoff $\delta_{\text {cut }}$ is not important.
} 
Eq. 22.8) illustrates the difference between $n(R)$ and $n_{S}(R)$. The latter has an expansion similar to eq.(2.8), but with the usual Taylor-expansion coefficients, 1 in front of $\alpha_{S}$ and $1 / 2$ in front of $\beta_{S}$. One therefore has

$$
n_{S}(R)=n(R)-\frac{1}{2} \alpha_{S} \ln \left(k_{0} R\right)+\frac{1}{3} \beta_{S} \ln ^{2}\left(k_{0} R\right)+\ldots .
$$

Setting $n_{S}\left(k_{0}\right)=1$ for simplicity, eq.2.10 implies $n_{S}(R)=2 n(R)-1$ for $\beta_{S}=0$, and $n_{S}(R)=3 n(R)-2$ for $\alpha_{S}=0$. We will compute the variance $\sigma(R)$, and hence the PBH fraction, for the former relation.

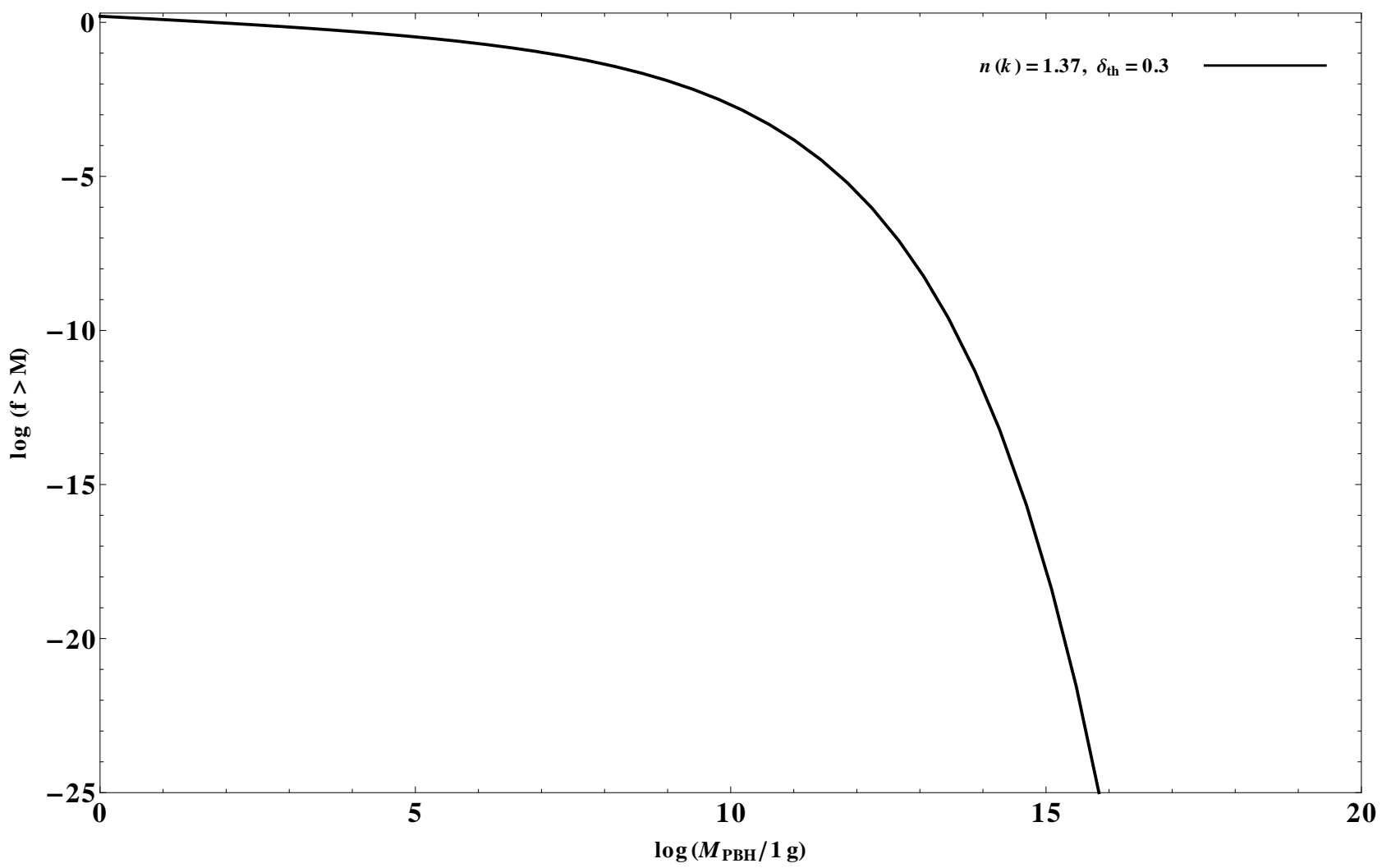

Figure 1: Fraction of the energy density of the universe collapsing into PBHs as a function of the PBH mass.

The result of this calculation is shown in figure 1. Here we have fixed $\gamma=0.2$, and show results for the threshold $\delta_{\text {th }}=1 / 3$. Due to Hawking radiation [25], PBHs contributing to Dark Matter (DM) today must have $M_{\mathrm{PBH}} \gtrsim 10^{15} \mathrm{~g}$; at this mass, they saturate the DM relic density if $f \simeq 5 \times 10^{-19}$ [26]. Figure 1 shows that this requires $n(R) \simeq 1.37$ for $\delta_{\text {th }}=0.3$. In order to get long-lived PBHs the amplitude of the perturbations at PBH scales must therefore exceed that at CMB scales by a factor $10^{3}-10^{4}$. Current data favor a negative or at best slightly positive value of $\alpha_{S}$ at the CMB pivot scale, as well as a spectral index at the pivot scale somewhat below 1. The first two terms in eq. (2.8) can thus not lead to PBH formation; so in the next section we study single-field inflation models by also considering the "running of the running of the spectral index" for PBHs formation. When possible, we will compare this to an exact calculation of the power of density perturbation at scales relevant for PBH formation. 


\section{Inflation Models and Primordial Black Holes}

Most models of inflation predict an approximately scale-free spectrum with a spectral index $n_{S}$ (as well as $n$ ) close to the scale-invariant (Harrison-Zel'dovich) case $n_{S}=n=1$. As shown, a significant number of long-lived PBHs can only be produced for $n>1$ (a "blue spectrum"), since these values lead to more power on small scales [6]. Observational limits (both from Hawking radiation and the fact that $\mathrm{PBHs}$ must not overclose the universe) strongly constrain $n_{S}$ [8]. This, therefore, yields a constraint on inflationary models that is independent of the cosmological constraints from the CMB and LSS. However, we will see below that in most simple models of inflation constraints on model parameters derived from the latter are far more stringent than the PBH constraint, to the point of making the formation of long-lived PBHs impossible.

A given inflation model can be described to lowest order in slow roll by three independent parameters: the normalization of the curvature perturbation spectrum $\mathcal{P}_{\mathcal{R}_{c}}$ at some scale, the tensor-to-scalar ratio $r$, and the spectral index $n_{S}$. If we wish to include higher-order effects, we have the forth and fifth parameters describing the running $\alpha_{S}$, and the running of running of the spectral index $\beta_{S}$.

Observational bounds on $\mathcal{P}_{\mathcal{R}_{c}}, r, n_{S}$ and $\alpha_{S}$ at the pivot $k_{\text {pivot }}=0.015 \mathrm{Mpc}^{-1}$, where $n_{S}$ and $\alpha_{S}$ are essentially uncorrelated, are reported in [11] as follows: : $^{5}$

$$
\begin{aligned}
n_{S}\left(k_{\text {pivot }}\right) & =0.9751 \pm 0.0110, \\
\alpha_{S}\left(k_{\text {pivot }}\right) & =-0.017 \pm 0.012, \\
\mathcal{P}_{\mathcal{R}_{c}}\left(k_{0}\right) & =(2.33 \pm 0.092) \times 10^{-9}, \\
r & <0.17(95 \% \mathrm{CL}) .
\end{aligned}
$$

By requiring $n_{S}(k) \in[0.9531,0.9971]$ and $\alpha_{S} \in[-0.041,0.007]$ for all $k \in\left[10^{-4}, 10\right] \mathrm{Mpc}^{-1}$ (i.e. down to the Lyman- $\alpha$ range), we find from eq.(2.8) that values of $\beta_{S}$ up to 0.017 are allowed. The ranges of $n_{S}$ and $\alpha_{S}$ are simply in $2 \sigma$ range of (3.1), and the range of $k$ encompasses all cosmologically relevant scales. Here we used the error bars derived from the analysis of the "SPT+WMAP7 $+\mathrm{BAO}+H_{0}+$ Clusters" data set 6 This upper bound on $\beta_{S}$ is lower than the bound found from the "WMAP $+H_{0}+\mathrm{BAO}$ " data set [7], since the older data set allowed somewhat larger values of $\alpha_{S}$. Note that the estimates for spectral index and its running are highly correlated for the typical COBE scale, $k_{0}=0.002 \mathrm{Mpc}^{-1}[27]$.

Eq.(2.8) shows that for $\alpha_{S}=0$ we only need $\beta_{S}\left(k_{0}\right) \simeq 0.0015$ in order to generate sufficiently large density perturbations to allow formation of $10^{15} \mathrm{~g}$ PBHs. Even if we set $\alpha_{S}\left(k_{0}\right)$ equal to its central value, $\alpha_{S}\left(k_{0}\right)=-0.017$, we only need $\beta_{S}\left(k_{0}\right) \simeq 0.0028$. In this model-independent analysis including the running of the running of the spectral index thus easily allows to ac-

\footnotetext{
${ }^{5}$ The amplitude of the primordial scalar fluctuations is reported at the "COBE" scale $k_{0}=0.002 \mathrm{Mpc}^{-1}$.

${ }^{6}$ We ignore possible tensor modes, which is appropriate for small-field models. Allowing a sizable contribution from tensor modes changes the mean value of $\alpha_{S}$ [10, but unfortunately the pivot scale where the spectral index and its running are uncorrelated is not reported in running+tensor model. In the SPT data, the inflation parameters are not reported in running+tensor model. So although in the large-field models, tensor modes are not negligible we will assume that the upper bound of $\beta_{S}$ is the same as in small-field models. Note that the precise value of the upper bound on $\beta_{S}$ derived here is not important for our analysis, since it is in any case well above the lower bound needed for successful PBH formation.
} 
commodate $\mathrm{PBH}$ formation in scenarios that reproduce all current cosmological observations at large scales.

Of course, this kind of model-independent analysis does not show whether simple, reasonably well-motivated inflationary models exist that can generate a sufficiently large $\beta_{S}$. In the following we study different models of inflation and check whether they can lead to PBHs formation. As a by-product, we also check whether these models can accommodate a sizably negative value of $\alpha_{S}$, as indicated by current data.

In order to calculate the spectral parameters $n_{S}, \alpha_{S}$ and $\beta_{S}$ defined in eqs. (2.9), we need the first four slow-roll parameters, defined as [2]:

$$
\begin{aligned}
\epsilon & \equiv \frac{M_{\mathrm{P}}^{2}}{2}\left(\frac{V^{\prime}}{V}\right)^{2}, \\
\eta & \equiv M_{\mathrm{P}}^{2} \frac{V^{\prime \prime}}{V}, \\
\xi^{2} & \equiv M_{\mathrm{P}}^{4} \frac{V^{\prime} V^{\prime \prime \prime}}{V^{2}}, \\
\sigma^{3} & \equiv M_{\mathrm{P}}^{6} \frac{V^{\prime 2} V^{\prime \prime \prime \prime}}{V^{3}}=2 M_{\mathrm{P}}^{4} \epsilon \frac{V^{\prime \prime \prime \prime}}{V} .
\end{aligned}
$$

Here $V(\phi)$ is the inflaton potential, $M_{\mathrm{P}}$ is the reduced Planck mass, and primes denote derivatives with respect to $\phi$. Note that $\epsilon$ is positive by definition whereas in spite of the square, $\xi^{2}$ can be either positive or negative. The square in $\xi^{2}$ and cube in $\sigma^{3}$ are to indicate that they are second and third-order in the slow-roll expansion, respectively. These parameters must be less than one for the slow-roll expansion to be valid. All these parameters are in general scaledependent, i.e. they have to be evaluated at the value of $\phi$ that the inflaton field had when the scale $k$ crossed out of the horizon. The spectral parameters are related to these slow-roll parameters by [2, 28]:

$$
\begin{aligned}
& n_{S}=1-6 \epsilon+2 \eta \\
& \alpha_{S}=-24 \epsilon^{2}+16 \epsilon \eta-2 \xi^{2} \\
& \beta_{S}=-192 \epsilon^{3}+192 \epsilon^{2} \eta-32 \epsilon \eta^{2}-24 \epsilon \xi^{2}+2 \eta \xi^{2}+2 \sigma^{3}
\end{aligned}
$$

In most (small-field) inflation models, eqs.(3.2) imply two strong inequalities between (combinations of) slow-roll parameters (hierarchy):

$$
\begin{aligned}
|\epsilon| & \ll|\eta|, \\
|\epsilon \eta| & \ll\left|\xi^{2}\right| .
\end{aligned}
$$

The first relation means that $n_{S}-1$ is essentially determined by $\eta$. Similarly, both relations together imply that $\alpha_{S}$ is basically fixed by $\xi^{2}$, while only the last two terms in the expression for $\beta_{S}$ are relevant; these two terms are generically of similar order of magnitude.

Along with these, another crucial inflationary observable is the influence of gravitational waves, relative to density perturbations, on large-angle microwave background anisotropies, given by [2]

$$
r \equiv \frac{C_{2}(\text { grav })}{C_{2}(\text { dens })} \simeq 14 \epsilon
$$


Combining these equations gives [29]

$$
\begin{aligned}
\alpha_{S} & \simeq 6\left(\frac{r}{7}\right)^{2}+4\left(\frac{r}{7}\right)\left(n_{S}-1\right)-2 \xi^{2} \\
\beta_{S} & \simeq-15\left(\frac{r}{7}\right)^{3}-15\left(\frac{r}{7}\right)^{2}\left(n_{S}-1\right)-2\left(\frac{r}{7}\right)\left(n_{S}-1\right)^{2} \\
& +\frac{\alpha_{S}}{2}\left[9\left(\frac{r}{7}\right)-\left(n_{S}-1\right)\right]+2 \sigma^{3}
\end{aligned}
$$
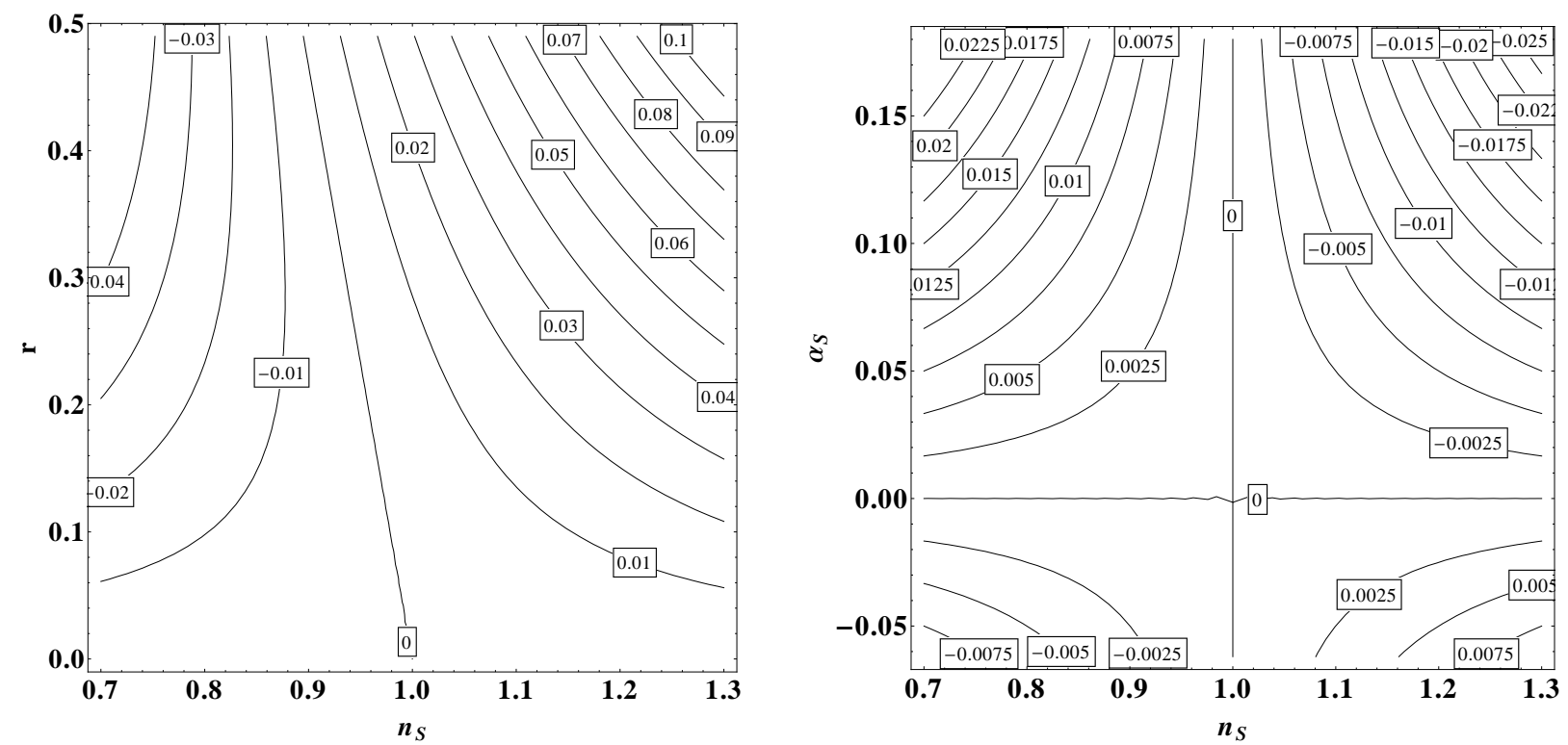

Figure 2: Contours of $\alpha_{S}+2 \xi^{2}$ (left) and of $\beta_{S}-2 \sigma^{3}$ (right); the right frame assumes negligible tensor modes, $r=0$.

In the left frame of figure 2 we show contours of $\alpha_{S}+2 \xi^{2}$ in the $\left(n_{s}, r\right)$ plane, while contours of $\beta_{S}-2 \sigma^{3}$ in the $\left(n_{S}, \alpha_{S}\right)$ plane are shown in the right frame, assuming a negligible tensorto-scalar ratio. We see that the observational constraints on $n_{S}$ and $r$ imply that $\alpha_{S}+2 \xi^{2}$ is very small, roughly $-9 \times 10^{-4} \leq \alpha_{S}+2 \xi^{2} \leq 2.2 \times 10^{-3}$ if $n_{S}$ and $r$ are within their current $2 \sigma$ intervals. Any significant running must therefore be due to $\xi^{2}\left[30\right.$. Similarly, $-5 \times 10^{-4} \leq$ $\beta_{S}-2 \sigma^{3} \leq-4 \times 10^{-5}$ if $r=0$ and $n_{S}$ and $\alpha_{S}$ are within their $1 \sigma$ intervals. Even using $2 \sigma$ intervals and allowing $r \leq 0.17$, this range only expands to $-0.0053 \leq \beta_{S}-2 \sigma^{3} \leq 0.001$, so that significant positive running of the running can only be due to $\sigma^{3}$.

In slow-roll approximation, the absolute normalization of the power spectrum is given by

$$
\mathcal{P}_{\mathcal{R}_{c}}=\frac{1}{12 \pi^{2} M_{\mathrm{P}}^{6}} \frac{V^{3}}{V^{\prime 2}} .
$$

Finally, the number of $e$-folds of slow-roll inflation that occurred from the time $t_{*}$ when observable CMB scales first crossed the Hubble radius during inflation to the epoch $t_{\text {end }}$ when inflation ended is given by

$$
N=\frac{1}{M_{\mathrm{P}}^{2}} \int_{\phi_{\mathrm{end}}}^{\phi_{*}} \frac{V}{V^{\prime}} d \phi .
$$


where $\phi_{\text {end }}$ is defined by $\max \left[\epsilon\left(\phi_{\text {end }}\right),\left|\eta\left(\phi_{\text {end }}\right)\right|\right]=1$; note that inflation might end through dynamics of other fields coupled to the inflaton, as in hybrid inflation. The observationally required value of $N$ depends logarithmically on the reheating temperature 7 Assuming instantaneous change from inflation to relativistic matter domination, a reasonable range of values of the number of $e$-folds between $t_{*}$ and $t_{\text {end }}$ is taken to be $N=54 \pm 7$ [31. Requiring baryogenesis to take place at or above the electroweak scale implies that $N \gtrsim 30$. A value of $N \simeq 60$ corresponds to a GUT scale reheating, 8

In the following we study the possibility of $\mathrm{PBH}$ formation in two different categories of inflation models: small-field models and large-field models. Hybrid models [32] are not studied here because in these models, PBH formation can occur by different mechanisms. (For analyses of PBH formation in hybrid models, see [33.) As noted above, the spectral index will have to increase at very small scales (very large $k$ ) in order to allow $\mathrm{PBH}$ formation, but we will also check whether the models we analyze are compatible with a sizably negative value of $\alpha_{S}$ at scales probed by the CMB and LSS data, as indicated by eqs.(3.1).

\subsection{Small-field models}

Small-field models are defined as those for which the variation in the inflaton field is less than the reduced Planck mass. Typically, $\epsilon$ and hence the amplitude of gravitational waves generated in such models is small and the spectral index and its running provide the key observational discriminators.

\subsubsection{Hilltop/inflection point inflation}

A popular ansatz for the small field inflaton potential is given by [3]

$$
V(\phi)=V_{0}\left[1-\left(\frac{\phi}{\mu}\right)^{p}\right],
$$

where $V_{0}, \mu$ and $p$ are positive constants ${ }^{9}$ This potential is equivalent to the potential $V(\phi)=$ $\Lambda^{4}-\lambda M_{\mathrm{P}}^{4-n} \frac{\phi^{n}}{n}$ in the literature [34] which can be specialized to several distinct models: e.g. hilltop $(n=2$ or $n=4)$ and inflection point $(n=3)$. We consider the case that the dominant term is the leading one, $V_{0}$. When $p$ is an integer and greater than 2 , such a potential may be generated by the self-coupling of the inflaton at tree-level.

For $p>0$, the hierarchies $(3.4)$ hold among slow-roll parameters. So the spectral parameters

\footnotetext{
${ }^{7}$ Instantaneous reheating gives the minimum number of $e$-folds as one looks backwards to the time of perturbation production, while a prolonged period of reheating gives a larger number of $e$-folds.

${ }^{8}$ Arbitrarily many $e$-folds of inflation might have occurred at $t<t_{*}$, as in "eternal" inflation. $N$ of eq. (3.8) is a lower bound on the total number of $e$-folds of inflation.

${ }^{9}$ This potential is unbounded from belove for $\phi \rightarrow \infty$. There must be additional terms that prevent this. Here we follow the usual assumption that these terms do not affect the dynamics of inflation.
} 
are given by:

$$
\begin{aligned}
n_{S}-1 & \simeq-2 p(p-1)\left(\frac{M_{\mathrm{P}}}{\mu}\right)^{2}\left(\frac{\phi}{\mu}\right)^{p-2} ; \\
\alpha_{S} & \simeq-2 p^{2}(p-1)(p-2)\left(\frac{M_{\mathrm{P}}}{\mu}\right)^{4}\left(\frac{\phi}{\mu}\right)^{2(p-2)} ; \\
\beta_{S} & \simeq-4 p^{3}(p-1)(p-2)^{2}\left(\frac{M_{\mathrm{P}}}{\mu}\right)^{6}\left(\frac{\phi}{\mu}\right)^{3(p-2)},
\end{aligned}
$$

Inflation ends at $\phi_{\text {end }} \lesssim \mu$, and in order to have a small field model we take $\mu \lesssim M_{\mathrm{P}}$. Then

$$
N=-\frac{p-1}{p-2}+\frac{1}{p(p-2)}\left(\frac{\mu}{M_{\mathrm{P}}}\right)^{2}\left(\frac{\mu}{\phi}\right)^{p-2} .
$$

For $p>2$, the first term in eq. 3.11) can be neglected. We then find, independently of $\mu$ :

$$
\begin{aligned}
n_{S}-1 & \simeq-\frac{p-1}{p-2} \frac{2}{N} \\
\alpha_{S} & \simeq-\frac{p-1}{p-2} \frac{2}{N^{2}}=\frac{1}{N}\left(n_{S}-1\right), \\
\beta_{S} & \simeq-\frac{p-1}{p-2} \frac{4}{N^{3}}=\frac{2}{N^{2}}\left(n_{S}-1\right) .
\end{aligned}
$$

In figure 3 the spectral index, its running and its running of running are shown as functions of the number of $e$-folds before the end of inflation, for $p=4$.

It is clear that in this model both $n_{S}-1$ and $\alpha_{S}$ are negative, but it is not possible to reproduce the observed central value of $\alpha_{S}$, which would require $\alpha_{S} \sim \mathcal{O}\left(n_{S}-1\right.$ ) (see figure 3 ). Moreover, the value of $\beta_{S}$ is also negative. So the conclusion is that this model cannot produce sufficient high density fluctuations at small scales to produce PBHs.

In the case at hand, the power spectrum can be calculated exactly as function of $N$. Again neglecting the first term in eq. (3.11) we have from eq.(3.7):

$$
\mathcal{P}_{\mathcal{R}_{c}}(N)=\frac{1}{12 \pi^{2}} \frac{V_{0} \mu^{2}}{p^{2} M_{\mathrm{P}}^{6}}\left[N p(p-2) \frac{M_{\mathrm{P}}^{2}}{\mu^{2}}\right]^{\frac{2 p-2}{p-2}} .
$$

Note that the exponent is positive for $p>2$. This implies less power at smaller $N$, i.e. at smaller length scales.

\subsubsection{Running mass inflation}

Another small-field model of interest is the running-mass model [35]. The possibility of PBHs formation in this model is studied in detail in [7]. This model is based on the inflationary potential

$$
V(\phi)=V_{0}+\frac{1}{2} m_{\phi}^{2}(\phi) \phi^{2},
$$

where $\phi$ is a real scalar. The potential (3.14) by itself would lead to eternal inflation, so again some terms need to be added, and we again assume that these terms do not affect the dynamics 


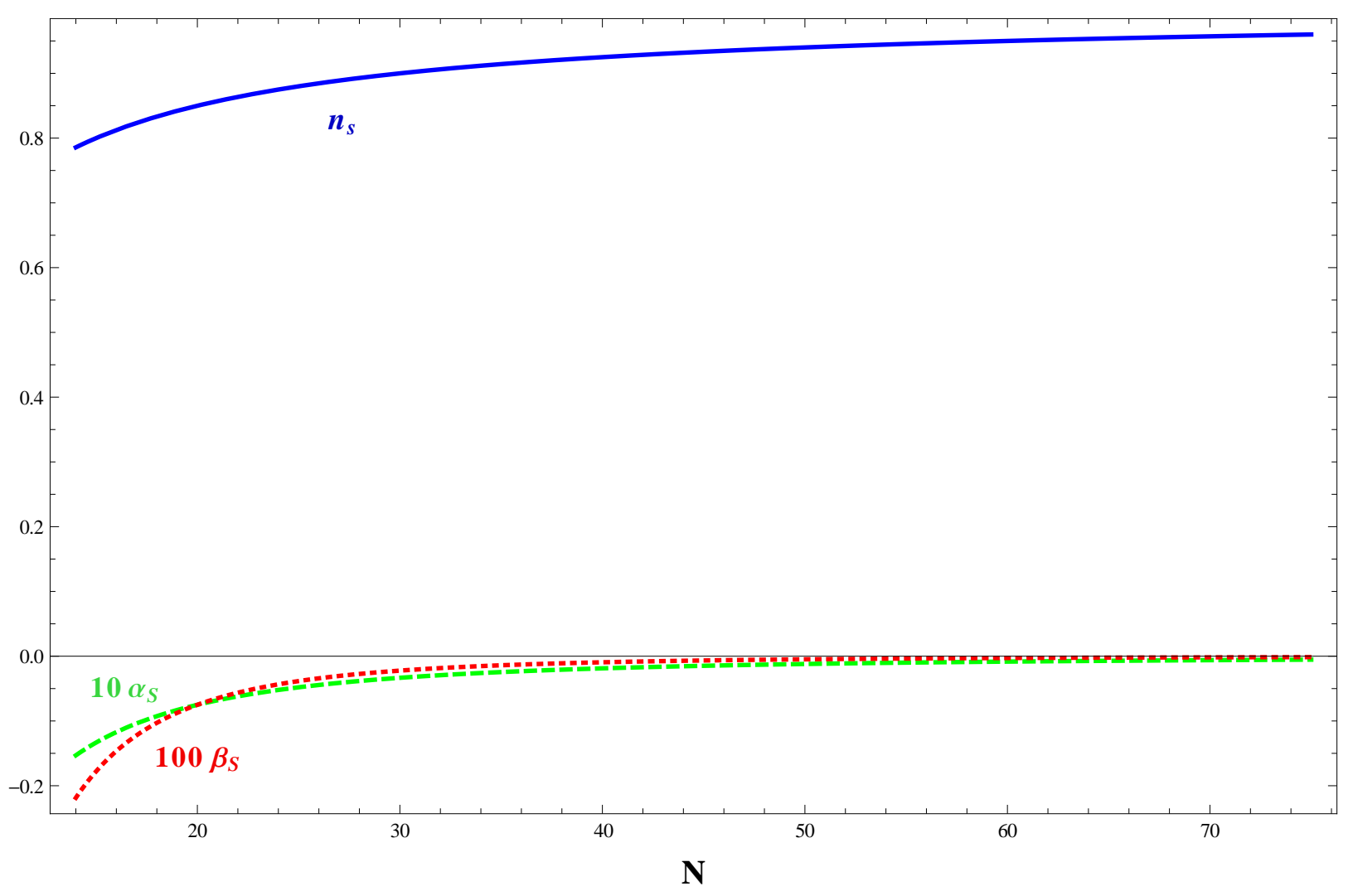

Figure 3: Illustrating the dependence according to eqs. (3.12) of $n_{S}$ (solid curve), $10 \alpha_{S}$ (dashed curve) and $100 \beta_{S}$ (dotted curve), on the number of $e$-folds before the end of inflation, for the fixed value of $p=4$.

of the inflaton during the slow-roll phase. During inflation, the potential is dominated by the constant term $V_{0}$. Here $m_{\phi}^{2}(\phi)$ is obtained by integrating a renormalization group equation of the form

$$
\frac{d m_{\phi}^{2}}{d \ln \phi} \equiv \beta_{m}=-\frac{2 C}{\pi} \alpha \widetilde{m}^{2}+\frac{D}{16 \pi^{2}}\left|\lambda_{Y}\right|^{2} m_{s}^{2}
$$

where $\beta_{m}$ is the $\beta$-function of the inflaton mass parameter which arises from the gauge interaction with coupling $\alpha$ and from the Yukawa interaction $\lambda_{Y} . C$ and $D$ are positive numbers of order one, which depend on the representations of the fields coupling to $\phi, \widetilde{m}$ is a gaugino mass parameter, while $m_{s}^{2}$ is the scalar SUSY breaking mass-squared of the scalar particles interacting with the inflaton via Yukawa interaction $\lambda_{Y}$.

Over a sufficiently small range of $\phi$, or small inflaton coupling, we can perform a Taylor expansion around $\phi_{*}$

$$
V=V_{0}+\frac{1}{2} m_{\phi}^{2}\left(\phi_{*}\right) \phi^{2}+\frac{1}{2} c \phi^{2} \ln \left(\frac{\phi}{\phi_{*}}\right)+\frac{1}{4} g \phi^{2} \ln ^{2}\left(\frac{\phi}{\phi_{*}}\right),
$$

where $\phi_{*}$ is the local extremum of the potential. Here $\left.c \equiv \frac{d m_{\phi}^{2}}{d \ln \phi}\right|_{\phi=\phi_{*}}$ is given by the $\beta$-function, 
and $\left.g \equiv \frac{d^{2} m_{\phi}^{2}}{d(\ln \phi)^{2}}\right|_{\phi=\phi_{*}}$ is given by the scale dependence of the parameters. By having the potential in hand and noting that the hierarchies (3.4) among slow-roll parameters hold in this model, we find the spectral parameters,

$$
\begin{aligned}
n_{S}-1 & =2 \frac{c M_{\mathrm{P}}^{2}}{V_{0}}\left[L+1+\frac{g}{2 c}\left(L^{2}+3 L+1\right)\right], \\
\alpha_{S} & =-2\left(\frac{c M_{\mathrm{P}}^{2}}{V_{0}}\right)^{2} L\left[1+\frac{g}{2 c}(2 L+3)\right]\left[1+\frac{g}{2 c}(L+1)\right], \\
\beta_{S} & =2\left(\frac{c M_{\mathrm{P}}^{2}}{V_{0}}\right)^{3} L\left[1+\frac{g}{2 c}(L+1)\right]\left[1+\frac{g}{2 c}(3 L+2)+\frac{g^{2}}{2 c^{2}}\left(3 L^{2}+5 L+\frac{3}{2}\right)\right],
\end{aligned}
$$

where $L \equiv \ln \frac{\phi}{\phi_{*}}$. Clearly the spectral index is not scale-invariant unless $c$ and $g$ are very close to zero.

An important feature of the running-mass model is that because of a consistency relation among the spectral index and its running, it is not possible [7] to get large negative running, which is presently favored by observation:

$$
\alpha_{S} \geq-\frac{\left(n_{S}-1\right)^{2}}{4} .
$$

On the other hand, we showed in [7] that over a narrow region of allowed parameter space this model predicts sufficiently large density perturbations at small scales to allow $10^{15} \mathrm{~g}$ PBHs to saturate the Dark Matter density.

\subsubsection{Inverse power law inflation}

A generic feature of models in nonperturbative gauge dynamics in SUSY [36] is the presence of scalar potentials of the form $\frac{\Lambda_{3}^{p+4}}{\phi^{p}}$, where the index $p$ and the scale $\Lambda_{3}$ depend on the underlying gauge group. Like models of hybrid inflation [32], these models are characterized by a potential dominated by the constant term $V_{0}$ and require coupling to another sector to end inflation when $\phi$ reaches the critical value $\phi_{c}$. Unlike standard hybrid inflation models, models of this type postulate a field far from the minimum of the potential.

We take the potential to be described by a single degree of freedom $\phi$, of the general form

$$
V(\phi)=V_{0}+\frac{\Lambda_{3}^{p+4}}{\phi^{p}}+\ldots
$$

where the dots represent nonrenormalizable terms suppressed by powers of the Planck mass, which are not relevant for the present discussion, but will prevent $\phi$ from "running away" to infinity. In the limit $\phi \ll\langle\phi\rangle$, the term $\sim \phi^{-p}$ dominates the dynamics:

$$
\begin{aligned}
V(\phi) & \simeq V_{0}+\frac{\Lambda_{3}^{p+4}}{\phi^{p}}, \quad \phi \ll\langle\phi\rangle \\
& =V_{0}\left[1+\alpha\left(\frac{M_{\mathrm{P}}}{\phi}\right)^{p}\right]
\end{aligned}
$$


where $\alpha \equiv \frac{\Lambda_{3}^{p+4}}{M_{\mathrm{P}}^{p} V_{0}}$. We assume that the constant $V_{0}$ dominates the potential, or $\alpha \ll\left(\phi / M_{\mathrm{P}}\right)^{p}$. In this case also hierarchies (3.4) hold among the slow-roll parameters which leads to the following spectral parameters:

$$
\begin{aligned}
n_{S}-1 & \simeq 2 p(p+1) \alpha\left(\frac{M_{\mathrm{P}}}{\phi}\right)^{p+2} ; \\
\alpha_{S} & \simeq-2 p^{2}(p+1)(p+2) \alpha^{2}\left(\frac{M_{\mathrm{P}}}{\phi}\right)^{2(p+2)} ; \\
\beta_{S} & \simeq 4 p^{3}(p+1)(p+2)^{2} \alpha^{3}\left(\frac{M_{\mathrm{P}}}{\phi}\right)^{3(p+2)} .
\end{aligned}
$$

The number of the $e-$ folds $N$ is given by

$$
N \simeq \frac{1}{p(p+2) \alpha}\left[\left(\frac{\phi_{c}}{M_{\mathrm{P}}}\right)^{p+2}-\left(\frac{\phi}{M_{\mathrm{P}}}\right)^{p+2}\right],
$$

where $\phi_{c}$ is the critical value at which inflation ends. The value of $\phi_{c}$ is in general determined by a coupling of the field to some other sector of the theory which we have here left unspecified. Note that from eq. 3.22 , for $\phi \ll \phi_{c}$ the number of $e$-folds approaches a constant, which we call $N_{\text {tot }}$,

$$
N_{\text {tot }} \equiv \frac{1}{p(p+2) \alpha}\left(\frac{\phi_{c}}{M_{\mathrm{P}}}\right)^{p+2} .
$$

This puts an upper limit on the total amount of expansion that takes place during the inflationary phase, although that upper bound can in principle be very large. Using eqs.(3.21)-(3.23), we can rewrite the cosmological parameters as functions of the number $N$ of $e$-folds before the end of inflation:

$$
\begin{aligned}
n_{S}-1 & \simeq \frac{p+1}{p+2} \frac{2}{N_{\text {tot }}\left(1-\frac{N}{N_{\text {tot }}}\right)}, \\
\alpha_{S} & \simeq-\frac{p+1}{p+2} \frac{2}{N_{\text {tot }}^{2}\left(1-\frac{N}{N_{\text {tot }}}\right)^{2}}=-\frac{p+2}{p+1} \frac{\left(n_{S}-1\right)^{2}}{2}, \\
\beta_{S} & \simeq \frac{p+1}{p+2} \frac{4}{N_{\text {tot }}^{3}\left(1-\frac{N}{N_{\text {tot }}}\right)^{3}}=\left(\frac{p+2}{p+1}\right)^{2} \frac{\left(n_{S}-1\right)^{3}}{2} .
\end{aligned}
$$

This model thus predicts $n_{S}>1$, which is currently disfavored at more than 2 standard deviation. Moreover, figure 4 shows that the spectrum becomes scale-invariant towards the end of inflation, i.e. it becomes less blue at smaller length scales, as also indicated by the negative value of $\alpha_{S}$. In combination with the constraint that $\left|n_{S}-1\right| \ll 1$ at CMB scale, this implies that this model cannot accommodate PBH formation. 


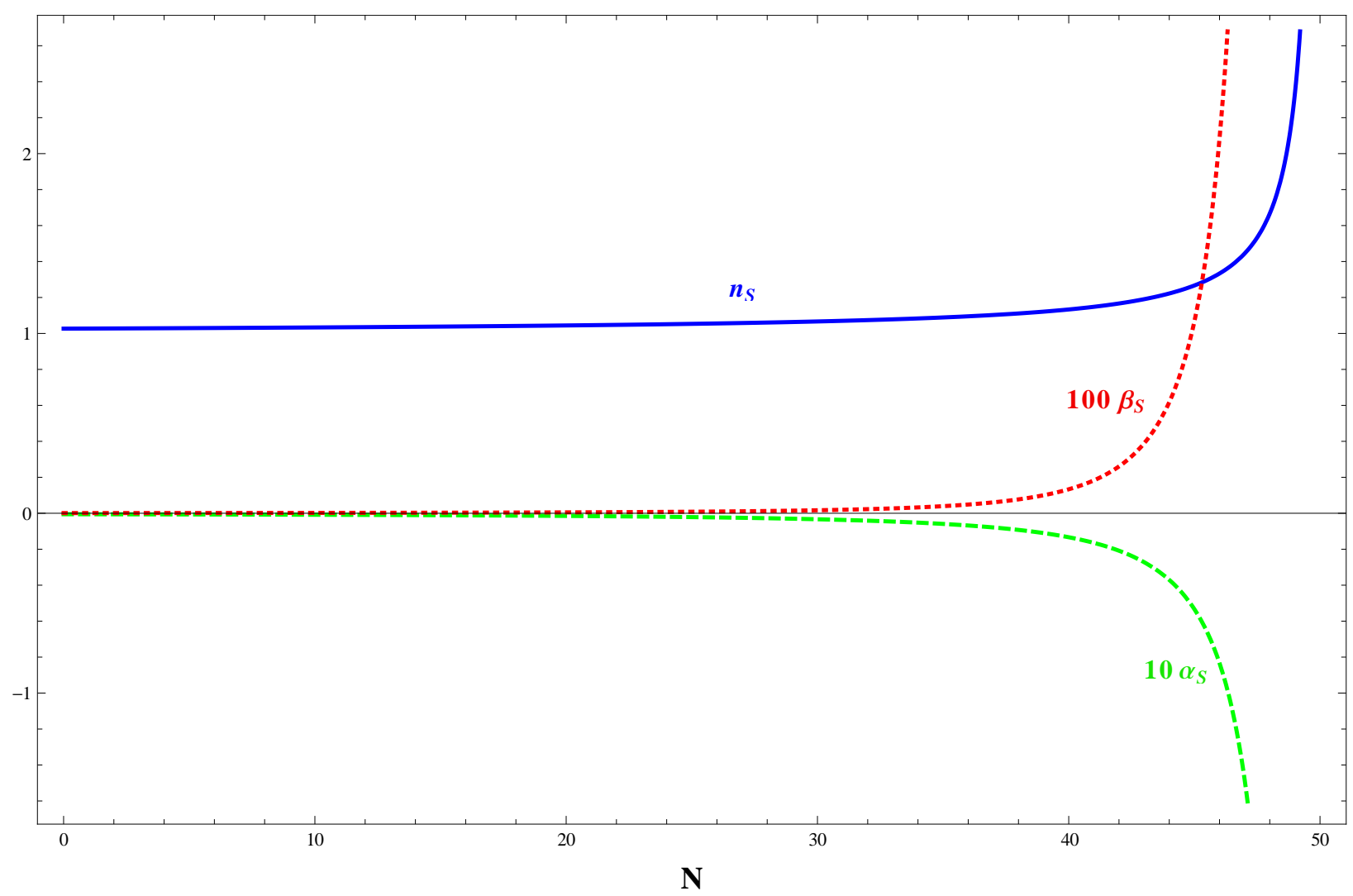

Figure 4: Spectral parameters as a function of the number of $e-$ folds $N \propto \ln (k)$ for $p=1$. Note especially the rapid approach to scale-invariance at short wavelengths $(\operatorname{small} N)$.

This can also be seen by directly computing the power spectrum as a function on $N$ :

$$
\mathcal{P}_{\mathcal{R}_{c}}(N)=\frac{V_{0}}{12 \pi^{2} M_{\mathrm{P}}^{4}} \frac{1}{\alpha^{2} p^{2}}\left[\alpha p(p+2)\left(N_{\text {tot }}-N\right)\right]^{\frac{2 p+2}{p+2}}
$$

where we have used (3.22). The power does increase with decreasing $N$, but only by a small amount. For example, the ratio of the power at the end of inflation $(N=0)$ to that at the COBE scale is

$$
q \equiv \frac{\mathcal{P}_{\mathcal{R}_{c}}(N=0)}{\mathcal{P}_{\mathcal{R}_{c}}\left(N_{\mathrm{COBE}}\right)}=\left(\frac{N_{\text {tot }}-N_{\mathrm{COBE}}}{N_{\text {tot }}}\right)^{-\frac{2 p+2}{p+2}} .
$$

On the other hand, the first eq. 3.24 gives

$$
n_{S}\left(N_{\mathrm{COBE}}\right)-1 \simeq \frac{p+1}{p+2} \frac{2}{N_{\mathrm{COBE}}\left(\frac{N_{\mathrm{tot}}}{N_{\mathrm{COBE}}}-1\right)} .
$$

Eq.(3.26) can be rewritten as

$$
\frac{1}{\frac{N_{\mathrm{tot}}}{N_{\mathrm{COBE}}}-1}=q^{\frac{p+2}{2 p+2}}-1
$$


Inserting this into eq. (3.27) finally yields

$$
q^{\frac{p+2}{2 p+2}}=1+\frac{p+2}{2(p+1)} N_{\mathrm{COBE}}\left[n_{S}\left(N_{\mathrm{COBE}}\right)-1\right] \simeq 2 .
$$

The power can therefore only increase by small amount in the course of inflation; in contrast, $\mathrm{PBH}$ formation would require an increase by a factor $10^{7}$ or so.

\subsection{Large-field models}

Large-field models are characterized by the condition $|\Delta \phi| \gtrsim M_{\mathrm{P}}$. Note that a super-Planckian field variation is a necessary condition for the generation of an observable tensor-to-scalar ratio [37. On the other hand, such large field models raise issues of stability in the presence of "quantum gravity" corrections, which are suppressed by inverse powers of $M_{\mathrm{P}}$. These corrections should not be important for small-field models, but need not be small for large-field models.

\subsubsection{Power-law (a.k.a. chaotic) inflation}

The polynomial potential $V(\phi)=\Lambda^{4}\left(\frac{\phi}{\mu}\right)^{p}$ is equivalent to $V(\phi)=\frac{\lambda}{M_{\mathrm{P}}^{p-4}} \phi^{p}$ in the literature [38]. In this model, the hierarchies (3.4) do not hold. We find:

$$
\begin{aligned}
n_{S}-1 & =-p(p+2)\left(\frac{M_{\mathrm{P}}}{\phi}\right)^{2} ; \\
\alpha_{S} & =-2 p^{2}(p+2)\left(\frac{M_{\mathrm{P}}}{\phi}\right)^{4} ; \\
\beta_{S} & =-8 p^{3}(p+2)\left(\frac{M_{\mathrm{P}}}{\phi}\right)^{6} ; \\
r & =7 p^{2}\left(\frac{M_{\mathrm{P}}}{\phi}\right)^{2} .
\end{aligned}
$$

Note that these quantities are independent of the normalization of the potential (described by $\Lambda^{4} / \mu^{p}$ or, equivalently, by $\lambda$ ), but do depend on its shape (described by $p$ ) as well as on the field value.

Inflation ends at $\phi_{\mathrm{end}}=\frac{p M_{\mathrm{P}}}{\sqrt{2}}$ where $\epsilon=1$. Then, it is straightforward to rewrite the inflation parameters as functions of the number of $e$-folds, $N$ :

$$
\begin{aligned}
n_{S}-1 & =-\frac{2(p+2)}{4 N+p}, \\
\alpha_{S} & =-\frac{8(p+2)}{(4 N+p)^{2}}=-\frac{2}{p+2}\left(n_{S}-1\right)^{2}, \\
\beta_{S} & =-\frac{64(p+2)}{(4 N+p)^{3}}=\frac{8}{(p+2)^{2}}\left(n_{S}-1\right)^{3}, \\
r & =\frac{14 p}{4 N+p}=-\frac{7 p}{p+2}\left(n_{S}-1\right) .
\end{aligned}
$$


Evidently the spectrum is "red" in this model, $n_{S}-1, \alpha_{S}$ and $\beta_{S}$ all being negative. However, this model also cannot accommodate the current central values, according to which both $\left|n_{S}-1\right|$ and $\left|\alpha_{S}\right|$ are of order $10^{-2}$. Moreover, $N_{\text {pivot }} \leq 50$ implies $n_{S}-1 \leq 0.040(0.059)$ for $p=2(4)$, i.e. $n_{S}-1$ comes out somewhat below the current central value in this model.

Computing the power directly from Eq.(3.7), we find:

$$
\mathcal{P}_{\mathcal{R}_{c}}(N)=\frac{1}{12 \pi^{2}}\left(\frac{\Lambda^{2}}{\mu M_{\mathrm{P}}}\right)^{2}\left[2 p\left(N+\frac{p}{4}\right)\right]^{\frac{p+2}{2}} .
$$

This decreases quickly towards the end of inflation $(N \rightarrow 0)$, again showing that PBH formation is not possible in this model.

\subsubsection{Generalized exponential inflation}

We now turn to the generalized exponential potential [39]:

$$
V(\phi)=\Lambda^{4} e^{(\phi / \mu)^{p}},
$$

where $p$ is a positive dimensionless constant and $\mu$ is a constant with dimension of mass. In this model the hierarchies (3.4) among the slow-roll parameters again do not hold and the values of the spectral parameters depend on the field value $\phi$ :

$$
\begin{aligned}
n_{S}-1 & =p\left(\frac{M_{\mathrm{P}}}{\mu}\right)^{2}\left[2(p-1)\left(\frac{\phi}{\mu}\right)^{p-2}-p\left(\frac{\phi}{\mu}\right)^{2 p-2}\right] ; \\
\alpha_{S} & =2 p^{2}(p-1)\left(\frac{M_{\mathrm{P}}}{\mu}\right)^{4}\left[p\left(\frac{\phi}{\mu}\right)^{3 p-4}-(p-2)\left(\frac{\phi}{\mu}\right)^{2 p-4}\right] ; \\
\beta_{S} & =2 p^{3}(p-1)\left(\frac{M_{\mathrm{P}}}{\mu}\right)^{6}\left[-p(3 p-4)\left(\frac{\phi}{\mu}\right)^{4 p-6}+2(p-2)^{2}\left(\frac{\phi}{\mu}\right)^{3 p-6}\right] ; \\
r & =7 p^{2}\left(\frac{M_{\mathrm{P}}}{\mu}\right)^{2}\left(\frac{\phi}{\mu}\right)^{2 p-2} .
\end{aligned}
$$

We allow $p$ to be a positive real (not necessarily integer) number. If $p$ is not integer, $\phi$ has to be non-negative to get a real potential. In any case the field $\phi$ will roll from larger to smaller values during inflation.

For $p>2$, both terms in the first eq. $(3.34)$, or equivalently both $\epsilon$ and $\eta$, decrease with decreasing $\phi$. The requirements $|\eta|<1, \epsilon<1$ then yield an upper bound on $\phi$, but inflation will never stop once $\phi$ is below this upper bound. This would require an additional mechanism to end inflation; we therefore only consider $p<2$ here.

For $p<2$, the requirement $|\eta|<1$ gives a lower bound on $\phi$, which is approximately given by

$$
\phi_{\min } \simeq \mu\left[\frac{p|p-1| M_{\mathrm{P}}^{2}}{\mu^{2}}\right]^{\frac{1}{2-p}} \quad(p<2) .
$$

This bound vanishes for $p=1$. Eqs.(3.34) show that this choice leads to a constant spectral index $n_{S}$ and vanishing $\alpha_{S}$ and $\beta_{S}$. This means that inflation does not end for $p=1$. Moreover, 


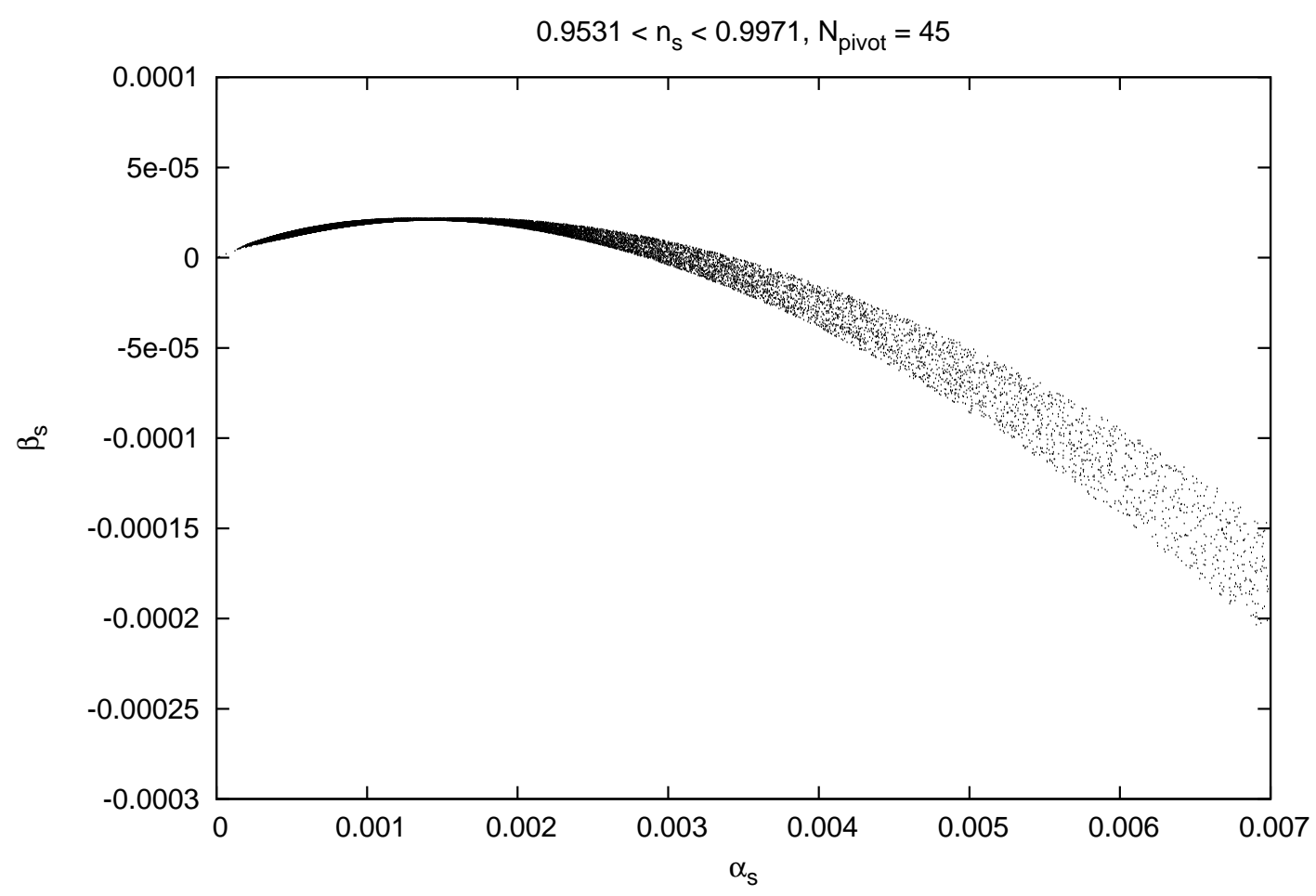

Figure 5: Scatter plot of allowed values of $\alpha_{S}$ and $\beta_{S}$ assuming that $n_{S}$ lies in its currently allowed $2 \sigma$ range and $45 e$-folds of inflation occurred after the pivot scale for potential (3.33).

$\alpha_{S}=0$ is (mildly) in conflict with present data, and a constant $n_{S}$ in the allowed range will not lead to $\mathrm{PBH}$ formation.

If $p>1$, the requirement $\epsilon<1$ implies an upper bound on $\phi$ :

$$
\phi_{\max } \simeq \mu\left(\frac{\mu}{p M_{\mathrm{P}}}\right)^{\frac{1}{p-1}} \quad(p>1) .
$$

The number of $e$-folds that occur after the inflaton field had a value $\phi$ is given by

$$
N(\phi)=\frac{1}{p(2-p)}\left(\frac{\mu}{M_{\mathrm{P}}}\right)^{2}\left[\left(\frac{\phi}{\mu}\right)^{2-p}-\left(\frac{\phi_{\min }}{\mu}\right)^{2-p}\right],
$$

where $\phi_{\min }$ is given by (3.35), and for $p>1, \phi$ has to satisfy $\phi<\phi_{\max }$, with $\phi_{\max }$ given by eq. (3.36).

For $p<1, n_{S}$ is always less than 1 , in accord with observation. The running of the spectral index, given by $\alpha_{S}$, is also negative, but we find $\alpha_{S}>-\left(n_{S}-1\right)^{2}$ for all allowed combinations of parameters that allow at least $30 e$-folds of inflation after the pivot scale. This model can therefore not accommodate a sizable and negative value of $\alpha_{S}$, either.

For $1<p<2, n_{S}-1$ can have either sign, while $\alpha_{S}$ is always positive, in contrast to the bound of current data. Quite large and positive $\alpha_{S}$ are in principle possible, if parameters are chosen such that the two contributions to $n_{S}-1$ in eq. 3.34 cancel approximately. However, such large values of $\alpha_{S}$ are definitely in conflict with observation. Moreover, if $\alpha_{S}>0.004$, $\beta_{S}$ turns negative, limiting the growth of power at small scales. This is illustrated in figure 5 , 
which shows a scatter plot of allowed values of $\alpha_{S}$ and $\beta_{S}$ assuming that $n_{S}$ lies in its currently allowed $2 \sigma$ range and $45 e$-folds of inflation occurred after the pivot scale.

Using the exact expression (3.7) we numerically find $n \leq 1.15$ at scales relevant for the formation of $10^{15} \mathrm{~g}$ PBHs; we saw in section 2 that $n>1.37$ is required for the formation of such PBHs. This model therefore cannot accommodate $\mathrm{PBH}$ formation, either.

\subsubsection{Inflation with negative exponential and Higgs inflation}

Another potential that has been proposed is [3]

$$
V(\phi)=V_{0}\left(1-e^{-q \phi / M_{\mathrm{P}}}\right) .
$$

For $q>0$ the inflaton field $\phi$ rolls towards smaller field values during inflation.10 The potential is sufficiently flat only for $q \phi>M_{\mathrm{P}}$, where the hierarchies (3.4) between slow-roll parameters hold. The inflationary parameters are

$$
\begin{aligned}
n_{S}-1 & \simeq-2 q^{2} e^{-q \phi / M_{\mathrm{P}}} \\
\alpha_{S} & \simeq-2 q^{4} e^{-2 q \phi / M_{\mathrm{P}}}=-\frac{1}{2}\left(n_{S}-1\right)^{2} ; \\
\beta_{S} & \simeq-4 q^{6} e^{-3 q \phi / M_{\mathrm{P}}}=\frac{1}{2}\left(n_{S}-1\right)^{3} ; \\
r & \simeq 7 q^{2} e^{-2 q \phi / M_{\mathrm{P}}},
\end{aligned}
$$

where we have approximated the denominators of eqs. (3.2) by $V_{0}$; this is appropriate for the phase of slow-roll where $n_{S} \simeq 1$, unless $q^{2} \ll 1$. Inflation ends at $\phi_{\text {end }}=2 M_{\mathrm{P}} \frac{\ln q}{q} . N e-$ folds before the end of inflation the spectral parameters are given by

$$
\begin{aligned}
n_{S}-1 & \simeq-\frac{2}{N+1} \\
\alpha_{S} & \simeq-\frac{2}{(N+1)^{2}} \\
\beta_{S} & \simeq-\frac{4}{(N+1)^{3}}, \\
r & \simeq \frac{7}{q^{2}} \frac{1}{(N+1)^{2}} .
\end{aligned}
$$

Note that the $q$-dependence cancels when the spectral parameters are expressed in terms of $N$.

In this model $n_{S}-1$ and $\alpha_{S}$ are manifestly negative, in agreement with current data. However, while $n_{S}-1$ also has approximately the right magnitude, $\left|\alpha_{S}\right|$ at the pivot scale is much smaller than the experimental central value. Moreover, since $\alpha_{S}$ and $\beta_{S}$ are both negative, $\mathrm{PBH}$ formation is not possible in this model; this can also be seen from the exact expression (3.7), which shows that the power always decreases with decreasing $N$.

\footnotetext{
${ }^{10}$ If negative values of $\phi$ are allowed, the potential 3.38 becomes unbounded for $\phi \rightarrow-\infty$. In this case additional terms have to be added to the potential, which we again assume to be unimportant during the slow-roll phase.
} 
A very similar potential describes the Higgs inflation model [40] where the Higgs boson of the Standard Model (SM) plays the role of the inflaton. Starting point of this model is the non-minimal coupling of the Higgs field to gravity. The relevant part of the action in the Jordan frame is:

$$
S_{\mathrm{J}}=\int \mathrm{d}^{4} x \sqrt{-g}\left\{-\frac{M^{2}+\xi h^{2}}{2} R+\frac{\partial_{\mu} h \partial^{\mu} h}{2}-\frac{\lambda}{4}\left(h^{2}-v^{2}\right)^{2}\right\},
$$

where $M$ is some mass parameter ${ }^{11}, R$ is the scalar curvature, $h$ is the Higgs field in the unitary gauge and $\xi$ determines the coupling of the Higgs to gravity ${ }^{12}$ By making a conformal transformation from the Jordan frame to the Einstein frame one can get rid of the non-minimal coupling:

$$
\hat{g}_{\mu \nu}=\Omega^{2} g_{\mu \nu}, \quad \Omega^{2}=1+\frac{\xi h^{2}}{M_{\mathrm{P}}^{2}} .
$$

This transformation induces a non-canonical kinetic energy term for $h$. It is therefore convenient to redefine $h$ in terms of the scalar field $\phi$ which casts the kinetic term into the canonical form [40]:

$$
\frac{d \phi}{d h}=\sqrt{\frac{\Omega^{2}+6 \xi^{2} h^{2} / M_{\mathrm{P}}^{2}}{\Omega^{4}}} .
$$

In terms of this new field, the potential is

$$
V(\phi)=\frac{1}{\Omega(\phi)^{4}} \frac{\lambda}{4}\left[h(\phi)^{2}-v^{2}\right]^{2} .
$$

For small field value, $h^{2} \ll M_{\mathrm{P}}^{2} / \xi$, one has $h \simeq \phi$ and $\Omega^{2} \simeq 1$; the two frames are indistinguishable so the potential for the field $\phi$ is the same as that for the initial Higgs field. However, for large values, $h \gg M_{\mathrm{P}} / \sqrt{\xi}$, one has $\Omega^{2} \simeq \xi h^{2} / M_{\mathrm{P}}^{2}$, and [40]

$$
h \simeq \frac{M_{\mathrm{P}}}{\sqrt{\xi}} \exp \left(\frac{\phi}{\sqrt{6} M_{\mathrm{P}}}\right) \text {. }
$$

Substituting this into eq. (3.46) we obtain the expression for the potential

$$
V(\phi)=\frac{\lambda M_{\mathrm{P}}^{4}}{4 \xi^{2}}\left[1-\exp \left(-\frac{2 \phi}{\sqrt{6} M_{\mathrm{P}}}\right)\right]^{2} .
$$

Recall that this expression holds only for $h \gg M_{\mathrm{P}} / \sqrt{\xi}$, which implies that the exponential term in eq. (3.48) is small. The square of this term is then even smaller, and can be neglected during inflation. The potential therefore effectively almost reduces to the form $(3.38)$, with $q=2 / \sqrt{6}$, except that the exponential term is multiplied with 2 (due to the square in eq.(3.48)). This also increases $n_{S}-1$ by a factor of 2 :

$$
n_{S}-1 \simeq-\frac{8}{3} \frac{M_{\mathrm{P}}^{2}}{\xi h^{2}} .
$$

\footnotetext{
${ }^{11}$ In the range of $\xi$ of interest to us, $M \simeq M_{\mathrm{P}}$ [0].

${ }^{12}$ Higgs inflation requires $\xi \gg 1$. This leads to a breakdown of tree-level unitarity at scales well below the Planck scale [41, but according to ref. 42] this does not invalidate the scenario, since the relevant energy scale during inflation always remains in the unitary regime.
} 
However, this factor of 2 cancels if $n_{S}-1$ is expressed in terms of the number of $e$-folds of inflation that occur after the field had the value $\phi$, i.e. the first eq. (3.40) remains valid in Higgs inflation. Moreover, $\alpha_{S}$ and $\beta_{S}$ are as in eqs. 3.39) when expressed in terms of $n_{S}-1$ or $N$. For most practical purposes (of inflation), Higgs inflation can therefore be understood as a particle physics implementation of negative exponential inflation.

\subsubsection{Natural inflation}

One way to obtain a very flat potential is to consider the natural inflation [43] where a Pseudo Nambu-Goldstone Boson (PNGB) is used as inflaton ${ }^{13}$ In this model, the inflaton field has a particular form of the potential which results from explicit breaking of a shift symmetry:

$$
V(\phi)=\Lambda^{4}\left[1 \pm \cos \left(a \frac{\phi}{f}\right)\right],
$$

We will take the positive sign in eq. (3.50) and assume that initially $\phi \ll f / a$. For appropriately chosen values of the mass scales, e.g. $f / a \sim M_{\mathrm{P}}$ and $\Lambda \sim M_{\mathrm{GUT}} \sim 10^{16} \mathrm{GeV}$, the PNGB field $\phi$ can drive inflation. We set $a=1$ for simplicity and treat $f$ as a free parameter. The Slow-roll parameters are then given by

$$
\begin{aligned}
\epsilon & =\frac{1}{2}\left(\frac{M_{\mathrm{P}}}{f}\right)^{2}\left[\frac{\sin (\phi / f)}{1+\cos (\phi / f)}\right]^{2} \simeq \frac{1}{8}\left(\frac{M_{\mathrm{P}}}{f}\right)^{2}\left(\frac{\phi}{f}\right)^{2} ; \\
\eta & =-\left(\frac{M_{\mathrm{P}}}{f}\right)^{2}\left[\frac{\cos (\phi / f)}{1+\cos (\phi / f)}\right]-\frac{1}{2}\left(\frac{M_{\mathrm{P}}}{f}\right)^{2} ; \\
\xi^{2} & =-\left(\frac{M_{\mathrm{P}}}{f}\right)^{4}\left[\frac{\sin (\phi / f)}{1+\cos (\phi / f)}\right]^{2} \simeq-\frac{1}{4}\left(\frac{M_{\mathrm{P}}}{f}\right)^{4}\left(\frac{\phi}{f}\right)^{2} ; \\
\sigma^{3} & =\left(\frac{M_{\mathrm{P}}}{f}\right)^{6} \frac{\cos (\phi / f) \sin ^{2}(\phi / f)}{[1+\cos (\phi / f)]^{3}} \simeq \frac{1}{8}\left(\frac{M_{\mathrm{P}}}{f}\right)^{6}\left(\frac{\phi}{f}\right)^{2},
\end{aligned}
$$

where the approximate equalities hold for $\phi \ll f$. It is clear that the hierarchies (3.4) do not hold among the slow-roll parameters ${ }^{14}$, and we find the following inflation parameters:

$$
\begin{aligned}
n_{S}-1 & =-\left(\frac{M_{\mathrm{P}}}{f}\right)^{2} \frac{3-\cos (\phi / f)}{1+\cos (\phi / f)} ; \\
\alpha_{S} & =-4\left(\frac{M_{\mathrm{P}}}{f}\right)^{4} \frac{1-\cos (\phi / f)}{[1+\cos (\phi / f)]^{2}} ; \\
\beta_{S} & =-4\left(\frac{M_{\mathrm{P}}}{f}\right)^{6} \frac{[1-\cos (\phi / f)][3-\cos (\phi / f)]}{[1+\cos (\phi / f)]^{3}} \\
r & =7\left(\frac{M_{\mathrm{P}}}{f}\right)^{2} \frac{1-\cos (\phi / f)}{1+\cos (\phi / f)}
\end{aligned}
$$

\footnotetext{
${ }^{13}$ Natural inflation can be either a large- or small-field inflation model, depending on the value of $f$. Here we assume $f>M_{\mathrm{P}}$.

${ }^{14}$ The first strong inequality in 3.4 does hold for $\phi \ll f$, but the second one does not even hold in this limit.
} 
Inflation ends at $|\eta|=1$ and the relation between the inflaton field and the number of $e$-folds is given by

$$
\cos \left(\frac{\phi}{f}\right)=1-y,
$$

where $y \equiv \frac{x^{2}+2}{x^{2}+1} e^{-N x^{2}}, x \equiv \frac{M_{\mathrm{P}}}{f}$. Inserting eq. 3.53 into 3.52 yields

$$
\begin{aligned}
n_{S}-1 & =-x^{2} \frac{2+y}{2-y}, \\
\alpha_{S} & =-4 x^{4} \frac{y}{(2-y)^{2}}, \\
\beta_{S} & =-4 x^{6} \frac{y(2+y)}{(2-y)^{3}}, \\
r & =7 x^{2} \frac{y}{2-y} .
\end{aligned}
$$

In the course of inflation $y$ increases from a rather small value to $y_{\text {end }}=\frac{2+x^{2}}{1+x^{2}}$ at the end of inflation $(N=0)$. Eqs. (3.54) show that $n_{S}-1, \alpha_{S}$ and $\beta_{S}$ become more negative as $y$ increases, indicating that the power is reduced at smaller scales. This can also be seen from the exact expression (3.7) which gives

$$
\mathcal{P}_{\mathcal{R}_{c}}=\frac{\Lambda^{4} f^{2}}{12 \pi^{2} M_{\mathrm{P}}^{6}} \frac{(2-y)^{2}}{y},
$$

which decreases with increasing $y \in[0,2]$. PBH formation is therefore not possible in this model.

For fixed $N$, the spectral parameters are determined by $x .\left|n_{S}-1\right|$ can clearly be made as large as desired (with $n_{S}<1$ ) by choosing a large value of $x$, i.e. a small value of $f$. On the other hand, $\left|\alpha_{S}\right|$ reaches a maximum at $x^{2} \simeq 2 / N$ for $y \ll 1$; note that $y$ decreases with increasing $x^{2}$. This gives $\alpha_{S} \gtrsim-1.5 / N^{2}$, i.e. $\left|\alpha_{S}\right|$ at the pivot scale cannot be larger than $10^{-3}$ in this model, well below the current central value.

\subsubsection{Arctan inflation}

Another inflation model which we are interested to study has been introduced in [44]:

$$
V(\phi)=V_{0}\left[1+\frac{2}{\pi} \arctan \left(\frac{\phi}{\mu}\right)\right] .
$$

This model allows inflation with $n_{S} \simeq 1$ if $\mu \gg M_{\mathrm{P}}$ or $\phi \gg \mu$. However, inflation can be ended by the potential (3.56) only if $\mu \lesssim 0.8 M_{\mathrm{P}}$, since otherwise $\epsilon,|\eta|<1 \quad \forall \phi$. A finite period of inflation thus requires that $\phi \gg \mu$ initially; at the end of inflation, $\phi \rightarrow-\infty$, i.e. $V \rightarrow 0$. During the slow-roll phase the hierarchies (3.4) between the slow-roll parameters hold, and we 
find

$$
\begin{aligned}
n_{S}-1 & \simeq-\frac{4}{\pi}\left(\frac{M_{\mathrm{P}}}{\phi}\right)^{2} \frac{\mu}{\phi} \\
\alpha_{S} & \simeq-\frac{12}{\pi^{2}}\left(\frac{M_{\mathrm{P}}}{\phi}\right)^{4}\left(\frac{\mu}{\phi}\right)^{2}=-\frac{3}{4}\left(n_{S}-1\right)^{2} \\
\beta_{S} & \simeq-\frac{72}{\pi^{3}}\left(\frac{M_{\mathrm{P}}}{\phi}\right)^{6}\left(\frac{\mu}{\phi}\right)^{3}=\frac{9}{8}\left(n_{S}-1\right)^{3} \\
r & \simeq \frac{7}{\pi^{2}}\left(\frac{M_{\mathrm{P}}}{\phi}\right)^{2}\left(\frac{\mu}{\phi}\right)^{2}
\end{aligned}
$$

where we have approximated the denominators of eqs. 3.2 by $2 V_{0}$, as appropriate for the slowroll phase where $\phi \gg \mu$. In terms of the number $N$ of $e$-folds of inflation that occurred after the inflaton field reached the value $\phi$, we find

$$
n_{S}-1 \simeq-\frac{4}{3 N+\pi},
$$

where we have used the fact that inflation ends at $\phi_{\text {end }} \simeq\left(M_{\mathrm{P}}^{2} \mu\right)^{1 / 3}$. This agrees with the currently allowed range for $38 \leq N \leq 95$. However, while $\alpha_{S}$ is negative, its absolute value is only of order $10^{-3}$ for allowed values of $n_{S}$; moreover, since $\beta_{S}$ is also negative, $\mathrm{PBH}$ formation is not possible. Indeed, one can see from the exact expression (3.7) that the power decreases steadily during inflation:

$$
\mathcal{P}_{\mathcal{R}_{c}}=\frac{V_{0}}{12 \pi^{2} M_{\mathrm{P}}^{6}}\left[1+\frac{2}{\phi} \arctan \left(\frac{\phi}{\mu}\right)\right]^{3}\left(1+\frac{\phi^{2}}{\mu^{2}}\right) .
$$

This decreases with decreasing $\phi$ for $\phi_{\text {end }} \leq \phi<\infty$.

\section{Summary and Conclusions}

In this paper we reviewed the formation of primordial black holes using the Press-Schechter formalism. We found that the formation of PBHs with mass larger than $10^{15} \mathrm{~g}$, whose lifetime exceeds the age of the Universe, will be produced at sufficient abundance to form the cold Dark Matter if the spectral index at scale $k_{\mathrm{PBH}}$ is about 1.37 for the threshold value $\delta_{\mathrm{th}}=1 / 3$. This spectral index is much above the value measured at much larger length scales in the CMB. $\mathrm{PBH}$ formation therefore requires significant positive running of the spectral index when $k$ is increased.

We compared this with the values of the spectral index and its running derived from current data on large scale structure. These include analyses of CMB anisotropies from the WMAP (7 year) and SPT collaborations, as well as data on baryonic acoustic oscillations and on the abundance of clusters, and direct measurements of the Hubble constant $H_{0}$. At the pivot scale of this data set one finds $n_{S}\left(k_{\text {pivot }}\right)=0.9751$ as central value. The first derivative $\alpha_{S}\left(k_{0}\right)$ would then need to exceed 0.020 if it alone were responsible for the required increase of the spectral index; this is more than $3 \sigma$ above the current central value of this quantity. However, the 
second derivative (the "running of the running") of the spectral index is currently only very weakly constrained. We showed in a model-independent analysis that this easily allows values of $n\left(k_{\mathrm{PBH}}\right)$ large enough for $\mathrm{PBH}$ formation, even if the first derivative of the spectral index is negative at CMB scales.

In section 3 we applied this formalism to a wide class of inflationary models, under the constraints imposed by the data mentioned above. We classified the inflation models in smallfield and large-field models. We have shown that only one small-field model, the running-mass model, allows sizable positive running of running of the spectral index, and is thus a good candidate for long-lived PBHs formation, albeit only in a narrow range of parameter space. In contrast, all the large-field models we studied predict small or negative values for the second derivative of the spectral index, and thus predict negligible $\mathrm{PBH}$ formation due to the collapse of overdense regions seeded during inflation. Recall, however, that other mechanisms of $\mathrm{PBH}$ formation have been suggested, e.g. in the "waterfall" phase of models of hybrid inflation, or during first order phase transitions.

As a by-product of our analysis, we found that most of the models we studied either predict $n_{S}<1$, as indicated by present data, or can at least accommodate it, the single exception being inverse power law inflation (a large field model). In contrast, none of the models we analyzed allows to reproduce a large negative value of $\alpha_{S}$, as preferred by current data; this confirms the results of the general analysis of ref.[45]. If future data confirm with high precision that $\alpha_{S} \lesssim-0.01$, all simple single-field models of inflation would be excluded. Similarly, proving conclusively that the second derivative of the spectral index is positive would exclude all the large-field models we investigated. Future analyses of the spectrum of primordial density perturbations thus hold great promise to discriminate between inflationary scenarios, or even to challenge the paradigm of single-field inflation.

\section{Acknowledgments}

This work was supported by the TR33 "The Dark Universe" funded by the Deutsche Forschungsgemeinschaft. EE also thanks the Bonn-Cologne Graduate School for support.

\section{References}

[1] For an introduction into the problems of standard cosmology and their solution by inflation, as well as a review of the early literature, see E. W. Kolb and M. S. Turner, The Early Universe, Addison-Wesley (1990).

[2] D. H. Lyth and A. R. Liddle, The Primordial Density Perturbation, Cambridge University Press (2009).

[3] For reviews on inflationary models, see D. Lyth and A. Riotto, Phys. Rept. 314 (1999) 1-146 [arXiv: hep-ph/9807278]; A. Mazumdar and J. Rocher, Phys. Rept. 497 (2011) 85 [arXiv: 1001.0993 [hep-ph]].

[4] B. J. Carr and S. W. Hawking, Mon. Not. Roy. Astron. Soc. 168 (1974) 399. 
[5] B. J. Carr, Astrophys. J. 201 (1975) 1.

[6] B. J. Carr and J. E. Lidsey, Phys. Rev. D48 (1993) 543; B. J. Carr, J. H. Gilbert and J. E. Lidsey Phys. Rev. D50 (1994) 4853 [arXiv: astro-ph/9405027].

[7] M. Drees and E. Erfani, JCAP 1104 (2011) 005 [arXiv: 1102.2340 [hep-ph]].

[8] A. M. Green and A. R. Liddle, Phys. Rev. D56 (1997) 6166 [arXiv: astro-ph/9704251]; H. I. Kim, C. H. Lee and J. H. MacGibbon, Phys. Rev. D59 (1999) 063004 [arXiv: astroph/9901030]; P. Pina, Phys. Rev. D72 (2005) 124004 [arXiv: astro-ph/0510052]; E. Bugaev and P. Klimai, Phys. Rev. D79 (2009) 103511 [arXiv: 0812.4247 [astro-ph]]; A. S. Josan, A. M. Green and K. A. Malik, Phys. Rev. D79 (2009) 103520 [arXiv: 0903.3184 [astroph.CO]]; K. Kohri, D. H. Lyth, A. Melchiorri, JCAP 0804 (2008) 038 [arXiv: 0711.5006 [hep-ph]]; L. Alabidi and K. Kohri, Phys. Rev. D80 (2009) 063511 [arXiv: 0906.1398 [astro-ph.CO]].

[9] D. H. Lyth, K. A. Malik, M. Sasaki and I. Zaballa, JCAP 0601 (2006) 011 [arXiv: astroph/0510647|; I. Zaballa, A. M. Green, K. A. Malik and M. Sasaki, JCAP 0703 (2007) 010 [arXiv: astro-ph/0612379].

[10] E. Komatsu et. al., Astrophys. J. Suppl. 192 (2011) 18 [arXiv: 1001.4538 [astro-ph.CO].

[11] R. Keisler et. al., arXiv: 1105.3182 [astro-ph.CO].

[12] W. J. Percival et al., MNRAS 401 (2010) 2148.

[13] A. G. Riess et al., ApJ 730 (2011) 119.

[14] A. Vikhlinin et. al., ApJ 692 (2009) 1060.

[15] W. H. Press and P. Schechter, Astrophys. J. 187 (1974) 425.

[16] Y. B . Zel'dovich and I. .D . Novikov, azh 43 (1966) 758; Y. B. Zel'dovich and I. D. Novikov, Sov. Astron. A. J. 10 (1967) 602.

[17] S. W. Hawking, Mon. Not. Roy. Astron. Soc. 152 (1971) 75.

[18] M. Y. Khlopov and A. G. Polnarev, Phys. Lett. B97 (1980) 383.

[19] S. W. Hawking, Phys. Lett. B231 (1989) 237.

[20] M. Crawford and D. N. Schramm, Nature 298 (1982) 538; I. G. Moss, Phys. Rev. D50 (1994) 676.

[21] S. G. Rubin, M. Y. Khlopov and A. S. Sakharov, Grav. Cosmol. 6 (2001) 1; V. I. Dokuchaev, Y. N. Eroshenko and S. G. Rubin, Grav. Cosmol. 11 (2005) 99 [arXiv: astro-ph/0412418.

[22] J. Niemeyer and K. Jedamzik, Phys. Rev. Lett. 80 (1998) 5481; Phys. Rev. D59 (1999) 124013. 
[23] A. S. Josan and A. M. Green, Phys. Rev. D82 (2010) 047303 [arXiv: 1004.5347 [hep-ph]].

[24] A. Kosowsky and M. S. Turner, Phys. Rev. D52 (1995) 1739; N. Düchting, Phys. Rev. D70 (2004) 064015 [arXiv: astro-ph/0406260]; Q.-G. Huang, JCAP 0611 (2006) 004 [arXiv: astro-ph/0610389.

[25] S. W. Hawking, Mon. Not. R. Astron. Soc. 168 (1974) 399.

[26] B. Carr, K. Kohri, Y. Sendouda and J. Yokoyama, Phys. Rev. D81 (2010) 104019 [arXiv: astro-ph/0912.5297].

[27] M. Cortês, A. R. Liddle and P. Mukherjee, Phys. Rev. D75 (2007) 083520 [arXiv: astroph/0702170.

[28] Q. Huang, JCAP 0611 (2006) 004 [arXiv: astro-ph/0610389].

[29] S. Hannestad, S. H. Hansen, F. L. Villante, Astropart. Phys. 16 (2001) 137 [arXiv: astroph/0012009.

[30] H. V. Peiris and R. Easther, JCAP 0807 (2008) 024 [arXiv: 0805.2154 [astro-ph]].

[31] A. R. Liddle and S. M. Leach, Phys. Rev. D68 (2003) 103503 [arXiv: astro-ph/0305263].

[32] A. D. Linde, Phys. Rev. D49 (1994) 748 [arXiv: astro-ph/9307002].

[33] D. H. Lyth, JCAP 1107 (2011) 035 [arXiv: 1012.4617 [astro-ph.CO]]; J. Garcia-Bellido, A. Linde and D. Wands, Phys. Rev. D54 (1996) 6040 [arXiv: astro-ph/9605094v3]; T. Kanazawa, M. Kawasaki and T. Yanagida, Phys. Lett. B482 (2000) 174 [arXiv: hepph/0002236v2].

[34] P. Adshead, R. Easther, J. Pritchard and A. Loeb, JCAP 1102 (2011) 021 [arXiv: 1007.3748v3 [astro-ph.CO]].

[35] E. D. Stewart, Phys. Lett. B391 (1997) 34 [arXiv: hep-ph/9606241]; E. D. Stewart, Phys. Rev. D56 (1997) 2019 [arXiv: hep-ph/9703232].

[36] G. F. Giudice, R. Rattazzi, Phys. Rept. 322 (1999) 419 [arXiv: hep-ph/9801271].

[37] D. H. Lyth, Phys. Rev. Lett. 78 (1997) 1861 [arXiv: hep-ph/9606387].

[38] A. D. Linde, Phys. Lett. B129 (1983) 177.

[39] A. Kosowsky and M. S. Turner, Phys. Rev. D52 (1995) 1739-1743 [arXiv: astro$\mathrm{ph} / 9504071 \mathrm{v} 2$ ].

[40] F. L. Bezrukov and M. Shaposhnikov, Phys. Lett. B659 (2008) 703 [arXiv: 0710.3755 [hep-th]].

[41] C. P. Burgess, H. M. Lee and M. Trott, JHEP 0909 (2009) 103 [arXiv: 0902.4465 [hep-ph]] and JHEP 1007 (2010) 007 [arXiv: 1002.2730 [hep-ph]]. 
[42] F. Bezrukov, A. Magnin, M. Shaposhnikov, S. Sibiryakov, JHEP 1101 (2011) 016 [arXiv: 1008.5157 [hep-ph]].

[43] K. Freese, J. A. Frieman and A. V. Olinto, Phys. Rev. Lett. 65 (1990) 3233.

[44] S. M. Leach, A. R. Liddle, J. Martin, D. J. Schwarz, Phys. Rev. D66 (2002) 023515 [arXiv: astro-ph/0202094.

[45] R. Easther and H. Peiris, JCAP 0609 (2006) 010 [arXiv: astro-ph/0604214]. 